\title{
DEPENDENCIES BETWEEN PIT-1 GENE POLYMORPHISM AND PRODUCTION PROGRESS IN POLISH HOLSTEIN-FRIESIAN BLACK-AND-WHITE COWS
}

\section{ZALEŻNOŚCI MIĘDZY POLIMORFIZMEM GENU PIT-1 A POSTĘPEM PRODUKCYJNYM U KRÓW RASY POLSKIEJ HOLSZTYŃSKO-FRYZYJSKIEJ ODMIANY CZARNO-BIAŁEJ}

\author{
Department of Animal Breeding and Product Quality Assessment, Poznań University \\ of Life Sciences, Poland \\ ${ }^{1}$ Computer Lab, Poznań University of Life Sciences, Poland \\ 2Department of Ruminant Science, West Pomeranian University of Technology, Szczecin, Poland
}

\begin{abstract}
Streszczenie. Celem badań była analiza zależności pomiędzy polimorfizmem genu PIT-1 w obrębie 6 eksonu pierwszego bydlęcego chromosomu (c.1178G>A) a wielkością postępu produkcyjnego u krów rasy polskiej holsztyńsko-fryzyjskiej odmiany czarno-białej. Stwierdzono, że największym rocznym postępem produkcyjnym i postępem kumulowanym w wydajności mleka i tłuszczu charakteryzowały się homozygoty AA. W przypadku wydajności białka w mleku korzystniejszymi wartościami obliczonych parametrów odznaczały się homozygoty GG.
\end{abstract}

Key words: cattle, polymorphism, PIT-1, production progress.

Słowa kluczowe: bydło, polimorfizm, PIT-1, postęp produkcyjny.

\section{INTRODUCTION}

Pituitary transcription factor PIT-1 (POU1F1) plays a significant role in developmental processes as an activator of the growth hormone gene [GH] (Bodner et al. 1988), the growth hormone releasing hormone receptor [GHRH-R] (Rhodes et al. 1993), prolactin [PRL] (Nelson et al. 1988) and the thyroid-stimulating hormone [TSH $\beta$ ] (Lin et al. 1994; Augustijn et al. 2002). In view of the multifaceted action of its product this gene has been selected as a candidate gene for studies on the relationships with numerous production traits, e.g. growth, slaughter performance traits and milk yield (Stancekova et al. 1999). Georges et al. (1995) and Woolard et al. (2000) reported that the POU1F1 locus on chromosome 1 (1q21-22) affects production traits in dairy cattle.

Results of studies conducted by various authors concerning dependencies between genetic variants of locus c.1178G $>A$ and production traits were inconclusive. Most probably it does not result from a lack of effect of this polymorphism on the function of the gene. For this

Corresponding author - Adres do korespondencji: Jarosław Pytlewski, Department of Animal Breeding and Product Quality Assessment, Poznań University of Life Sciences, Złotniki, Słoneczna 1, 62-002 Suchy Las, Poland, e-mail: jarekpyt@up.poznan.pl 
reason analyses presented in this paper may provide novel information on the relationship between genotypes at locus c.1178G $>A$ and production progress in Polish Holstein-Friesian Black-and-White cows. It is most advantageous to obtain considerable production progress within a short period of time with no deterioration of functional traits in cows and no increase in financial outlays on milk production. Atashl et al. (2006) suggested that it is possible to simultaneously improve lactation persistency and milk yield. Finding a respective genetic marker to reach this breeding objective within a short time would be highly advantageous for dairy cattle breeders and milk producers.

Literature sources lack results concerning a relationship between genetic variants at locus c. $1178 \mathrm{G}>A$ and the level of production progress in Polish Holstein-Friesian Black-and-White cows. In view of the practical aspect of this problem and a lack of studies on the subject it was decided to undertake research to realise the proposed objective.

The aim of this study was to analyse the dependence between polymorphism of the PIT-1 gene within exon 6 of bovine chromosome 1 and the level of production progress in Polish Holstein-Friesian Black-and-White cows.

\section{MATERIAL AND METHODS}

Analyses were conducted on 1099 Polish Holstein-Friesian Black-and-White cows (PHF). Cows were kept on six farms in the Wielkopolska region (Poland).

The study comprised the analysis of the polymorphic site $(c .1178 G>A)$ in gene PIT-1 located at chromosome 1 in exon 6 ( $G \rightarrow$ A mutation).

Analyses were conducted using PCR-RFLP.

The experiment consisted of the following stages:

a) isolation of genetic material - DNA (from peripheral blood) using phenol extraction;

b) amplification of a DNA fragment (451 bp) - conducted using a TGradient thermocycler (Biometra). Primer sequences provided by Woollard et al. (1994) were applied: Pit-1E6F-(5'-AAACCATCATCTCCCTTCTT-3'), Pit-1E6R-(5'-AATGTACAATGTCCTTCTGAG-3'). The reaction mixture of $15 \mu$ l contained $100 \mathrm{ng}$ genomic DNA, $0.6 \cup$ Taq polymerase, 10 pmol of each primer, $1.5 \mathrm{mM} \mathrm{MgCl} 2,200 \mu \mathrm{M}$ dNTP, $1.5 \mu \mathrm{l}$ PCR buffer $-\left(\mathrm{NH}_{4}\right)_{2} \mathrm{SO}_{4}$ (10x) and $0.75 \mu \mathrm{LMSO}$. Preliminary denaturation $\left(97^{\circ} \mathrm{C} / 300 \mathrm{sec}\right)$ was followed by 30 cycles of denaturation $\left(95^{\circ} \mathrm{C} / 30 \mathrm{sec}\right)$, primer annealing $\left(51^{\circ} \mathrm{C} / 30 \mathrm{sec}\right)$ and synthesis $\left(72^{\circ} \mathrm{C} / 40 \mathrm{sec}\right)$, completed with final synthesis $\left(72^{\circ} \mathrm{C} / 300 \mathrm{sec}\right)$;

c) digestion of PCR products using the restriction enzyme. Amplification products were digested at $37^{\circ} \mathrm{C}$ for $3 \mathrm{~h}$ using the restriction enzyme (Hinfl) and $\mathrm{R}$ buffer. The composition of the reaction mixture $(11 \mu \mathrm{l})$ for one sample was as follows: $5 \mu \mathrm{l} \mathrm{PCR}$ product, $1 \mu \mathrm{l}$ restriction enzyme at $10 \mathrm{U} / \mu \mathrm{l}$ (Fermentas), $1 \mu$ l enzymatic buffer (Fermentas) and $4 \mu \mathrm{l} \mathrm{H}_{2} \mathrm{O}$; d) verification of digestion products by agarose gel electrophoresis. Following digestion with restriction enzymes each sample was supplemented with $2 \mu$ loading buffer (Gel Loading Solution type I) $6 x$. Afterwards digestion products were verified by electrophoresis in $3 \%$ agarose gel (BASICA GQT, Prona) in $1 \times$ TBE buffer. The DNA Gene Ruler ${ }^{\mathrm{TM}}$ marker was used in the DNA Ladder Mix composed of $2 \mu$ loading buffer, $1.5 \mu$ DNA marker and $10.5 \mu \mathrm{l} \mathrm{H}_{2} \mathrm{O}$. The following settings were applied: electrophoresis time of $30 \mathrm{~min}$ and electric current of 140V. Digestion products were observed in UV light; 
e) genotype identification. The amplified fragment of the PIT-1 gene was $451 \mathrm{bp}$ in length. The following genotypes were identified: homozygote AA (-,-) - 451 bp (no site identified by the Hinfl restriction enzyme), heterozygote AG $(-,+)$ - fragments of 451,244 and $207 \mathrm{bp}$, and heterozygote GG $(+,+)$ - fragments of 244 and $207 \mathrm{bp}$;

f) statistical analysis. Results of laboratory analyses were subjected to the Hardy-Weinberg theorem. The $\mathrm{X}^{2}$ test was applied in statistical calculations. The frequency of alleles $A$ and $G$ at locus $c .1178 G>A$ was 0.1965 and 0.8035 , respectively. In turn, the observed genotype frequency was: $A A-0.0446, A G-0.3039$ and $G G-0.6515$. The applied $X^{2}$ test showed genetic equilibrium in the analysed population of cows.

Cows came from herds with average milk yields in 305-day lactation ranging from 5000 to $8000 \mathrm{~kg}$ milk. The animals were in their 1st to 10th lactation. Data on 3365 305-day lactations were collected from milk recording documentation from the period of 2002-2009. The shares of successive lactations in their total number were as follows: $1-28.4 \%$, $2-25.5 \%, 3-20.1 \%, 4-13.4 \%, 5-7.0 \%, 6-3.3 \%, 7-1.5 \%, 8-0.5 \%, 9-0.2 \%$ and $10-0.1 \%$, respectively. Milk yield and yields of milk fat and protein were recorded for each cow.

In this study annual production progress was calculated for the period of 2002-2009 along with cumulative progress for milk yield and yields of fat and protein for the entire analysed cattle population and in terms of division into genotypes at locus c.1178G $>A$.

Statistical analyses were performed using the following linear model:

$Y_{\mathrm{ijklmnop}}=\mu+H_{\mathrm{i}}+R_{\mathrm{j}}+S_{\mathrm{k}}+L_{1}+G_{\mathrm{m}}+\beta_{1} h f n+\beta_{2} w c O+e_{\mathrm{ijklmnop}}$ where:

$Y_{\mathrm{ijk} \text { Imnop }}$ - phenotypic value of analysed trait,

$\mu$ - population mean,

$H_{\mathrm{i}}$ - fixed effect of herd $(\mathrm{i}=1, \ldots, 6)$,

$R_{\mathrm{j}}$ - fixed effect of year of calving $(\mathrm{j}=1, \ldots, 8)$,

$S_{k}-$ fixed effect of season of calving $(k=1, \ldots, 4)$,

$L_{1}-$ fixed effect of lactation rank $(L=1, \ldots, 10)$,

$G_{m}$ - fixed effect of cow's genotype at locus c.1178G $>A(I=1, \ldots, 3)$,

$\beta_{1}, \beta_{2}-$ partial first-order linear regression coefficients:

hfn - share of HF genes in the genotype,

wco - age at first calving in days,

$e_{\text {ijklmnop }}$ - random residual effect.

Statistical calculations were performed using the SAS ${ }^{8}$ statistical package (2011) with the MEANS and GLM procedures.

The Duncan multiple range test was applied to compare object means.

\section{RESULTS}

Table 1 presents calculated values of annual production progress and cumulative progress for milk yields in the years 2002-2009 for the entire population of cows and in terms of division into genotypes at locus c.1178G $>A$. 
Table 1. Production progress for milk yield [kg] in the years 2002-2009 in the whole studied population of Holstein-Friesian Black-and-White cows ${ }^{a}$ and with regard to genotyping in locus c.1178G>A

Tabela 1. Postęp produkcyjny dla wydajności mleka [kg] w latach 2002-2009 w całej badanej populacji krów rasy polskiej holsztyńsko-fryzyjskiej odmiany czarno-białeja ${ }^{a}$ z uwzględnieniem podziału na genotypy w locus c.1178G $>A$

\begin{tabular}{|c|c|c|c|c|c|c|c|c|c|c|c|c|c|c|c|c|c|c|c|c|}
\hline \multirow{3}{*}{$\begin{array}{c}\text { Year } \\
\text { Rok } \\
* *\end{array}$} & \multirow{2}{*}{\multicolumn{5}{|c|}{$\begin{array}{l}\text { Analysed population } \\
\text { Badana populacja }\end{array}$}} & \multicolumn{15}{|c|}{ Genotype - in locus c. $1178 G>A-$ Genotyp w locus c. $1178 G>A$} \\
\hline & & & & & & \multicolumn{5}{|c|}{ AA $* *$} & \multicolumn{5}{|c|}{$A G * *$} & \multicolumn{5}{|c|}{ GG ** } \\
\hline & $\bar{x}$ & $\mathrm{x}_{\mathrm{i}}-\mathrm{x}_{\mathrm{i}-1}$ & $\%$ & $\mathrm{x}_{\mathrm{i}}-\mathrm{x}_{0}$ & $\%$ & $\bar{x}$ & $x_{i}-x_{i-1}$ & $\%$ & $\mathrm{x}_{\mathrm{i}}-\mathrm{x}_{0}$ & $\%$ & $\bar{x}$ & $x_{i}-x_{i-1}$ & $\%$ & $\mathrm{x}_{\mathrm{i}}-\mathrm{x}_{0}$ & $\%$ & $\bar{x}$ & $x_{i}-x_{i-1}$ & $\%$ & $\mathrm{x}_{\mathrm{i}}-\mathrm{x}_{0}$ & $\%$ \\
\hline 2002 & 5795.0 & A & & $\begin{array}{c}\text { ABCD } \\
\text { EFG }\end{array}$ & & 5531.9 & A & & $\begin{array}{c}\text { ABCD } \\
\text { EFG }\end{array}$ & & 5843.8 & & & $\begin{array}{l}\text { ABC } \\
\text { DEF }\end{array}$ & & 5794.7 & $\mathrm{~A}$ & & $\begin{array}{c}\text { ABCD } \\
\text { EFG }\end{array}$ & \\
\hline 2003 & 6105.4 & $\begin{array}{c}310.4 \\
A B \\
\end{array}$ & 105.4 & $\begin{array}{c}310.4 \\
\mathrm{~A} \\
\end{array}$ & 105.4 & 6086.0 & $\begin{array}{c}554.1 \\
\text { A }\end{array}$ & 110.0 & $\begin{array}{c}554.1 \\
\text { A }\end{array}$ & 110.0 & 5954.4 & $\begin{array}{c}110.7 \\
\mathrm{a}\end{array}$ & 101.9 & 110.7 & 101.9 & 6168.8 & $\begin{array}{c}374.1 \\
A B\end{array}$ & 106.5 & $\begin{array}{c}374.1 \\
\text { A }\end{array}$ & 106.5 \\
\hline 2004 & 6534.1 & $\begin{array}{c}428.7 \\
\text { BC }\end{array}$ & 107.0 & $\begin{array}{c}739.1 \\
\text { B } \\
\end{array}$ & 101.3 & 6619.8 & $\begin{array}{c}533.8 \\
\text { B }\end{array}$ & 108.8 & $\begin{array}{c}1087.9 \\
\text { B }\end{array}$ & 119.7 & 6421.5 & $\begin{array}{c}467.1 \\
\mathrm{a}\end{array}$ & 107.8 & $\begin{array}{c}577.7 \\
\text { A }\end{array}$ & 109.9 & 6577.4 & $\begin{array}{c}408.7 \\
\text { BC }\end{array}$ & 106.6 & $\begin{array}{c}782.8 \\
\text { B }\end{array}$ & 113.5 \\
\hline 2005 & 7058.0 & $\begin{array}{c}523.9 \\
\mathrm{C}\end{array}$ & 108.0 & $\begin{array}{c}1263.0 \\
C\end{array}$ & 121.8 & 6945.8 & $\begin{array}{c}326.0 \\
\text { B }\end{array}$ & 104.9 & $\begin{array}{c}1413.9 \\
C\end{array}$ & 125.6 & 6827.3 & 405.8 & 106.3 & $\begin{array}{c}983.5 \\
\text { B }\end{array}$ & 116.8 & 7167.3 & $\begin{array}{c}589.8 \\
\mathrm{C}\end{array}$ & 109.0 & $\begin{array}{c}1372.6 \\
C\end{array}$ & 123.7 \\
\hline 2006 & 7141.1 & $\begin{array}{c}83.1 \\
D\end{array}$ & 101.2 & $\begin{array}{c}1346.1 \\
D\end{array}$ & 123.2 & 7258.0 & $\begin{array}{c}312.2 \\
\mathrm{C}\end{array}$ & 104.5 & $\begin{array}{c}1726.1 \\
\mathrm{D}\end{array}$ & 131.2 & 7122.6 & $\begin{array}{c}295.3 \\
\text { A }\end{array}$ & 104.3 & $\begin{array}{c}1278.8 \\
\mathrm{C}\end{array}$ & 121.9 & 7143.0 & $\begin{array}{c}-24.3 \\
\text { D }\end{array}$ & 99.7 & $\begin{array}{c}1348.3 \\
D\end{array}$ & 123.3 \\
\hline 2007 & 7607.1 & $\begin{array}{c}466.0 \\
D E\end{array}$ & 106.5 & $\begin{array}{c}1812.1 \\
\mathrm{E}\end{array}$ & 131.3 & $\begin{array}{c}7180.5 \\
a b\end{array}$ & $\begin{array}{c}-77.5 \\
C D\end{array}$ & 98.9 & $\begin{array}{c}1648.6 \\
E\end{array}$ & 129.8 & $\begin{array}{c}7689.5 \\
a \\
\end{array}$ & $\begin{array}{c}567.0 \\
\text { A }\end{array}$ & 108.0 & $\begin{array}{c}1845.7 \\
\text { D }\end{array}$ & 131.6 & $\begin{array}{c}7589.1 \\
\mathrm{~b}\end{array}$ & $\begin{array}{c}446.2 \\
D E\end{array}$ & 106.2 & $\begin{array}{c}1794.5 \\
\text { E }\end{array}$ & 131.0 \\
\hline 2008 & 8125.2 & $\begin{array}{c}518.1 \\
\mathrm{E}\end{array}$ & 106.8 & $\begin{array}{c}2330.2 \\
\mathrm{~F} \\
\end{array}$ & 140.2 & $\begin{array}{c}7649.2 \\
A B\end{array}$ & $\begin{array}{c}468.7 \\
\text { DE }\end{array}$ & 106.5 & $\begin{array}{c}2117.3 \\
F\end{array}$ & 138.3 & $\begin{array}{c}8076.5 \\
\text { A }\end{array}$ & 387.0 & 105.0 & $\begin{array}{c}2232.7 \\
E\end{array}$ & 138.2 & $\begin{array}{c}8192.7 \\
\text { B }\end{array}$ & $\begin{array}{c}603.6 \\
\mathrm{E}\end{array}$ & 108.0 & $\begin{array}{c}2398.0 \\
F\end{array}$ & 141.4 \\
\hline 2009 & 8230.0 & 104.8 & 101.3 & $\begin{array}{c}2435.0 \\
\mathrm{G}\end{array}$ & 142.0 & 8158.2 & $\begin{array}{c}509.0 \\
\mathrm{E}\end{array}$ & 106.7 & $\begin{array}{c}2626.3 \\
G\end{array}$ & 147.5 & 8339.1 & 262.6 & 103.3 & $\begin{array}{c}2495.3 \\
F\end{array}$ & 142.7 & 8186.4 & -6.3 & 99.9 & $\begin{array}{c}2391.8 \\
G\end{array}$ & 141.3 \\
\hline $\bar{x}$ & & 347.9 & & & & & 375.2 & & & & & 356.5 & & & & & 341.7 & & & \\
\hline
\end{tabular}

$\mathrm{x}_{\mathrm{i}}-\mathrm{x}_{\mathrm{i}-1}$ - annual production progress - roczny postęp produkcyjny; $\mathrm{x}_{\mathrm{i}}-\mathrm{x}_{0}$ - production progress in relation to the initial year (cumulative production progress) - postęp produkcyjny w stosunku do roku początkowego (kumulowany postęp produkcyjny); ** statistically significant effect at $\mathrm{P} \leq 0.01-$ wpływ statystycznie istotny przy $\mathrm{P} \leq 0,01$. Means marked with the same letters are statistically different - Srednie oznaczone tymi samymi literami różnią się statystycznie: $A, B, C-$ at $P \leq 0.01-$ przy $P \leq 0,01$; $a$, b, $c-$ at $P \leq 0.05-$ przy $P \leq 0.05$; with $\mathrm{x}_{\mathrm{i}}-\mathrm{x}_{\mathrm{i}-1}$ differences between years are marked - przy $\mathrm{x}_{\mathrm{i}}-\mathrm{x}_{\mathrm{i}-1}$ oznaczono różnice między latami; for $\mathrm{x}_{\mathrm{i}}-\mathrm{x}_{0}$, differences are indicated in relation to the initial year within the group - przy $\mathrm{x}_{i}-\mathrm{x}_{0}$ oznaczono różnice w stosunku do roku początkowego w obrębie grupy; mean differences between individual genotypes in locus c.1178G> A were determined - przy średnich oznaczono różnice między poszczególnymi genotypami w locus c.1178G>A.

${ }^{a}$ until 2005 the Black-and-White name was used - do 2005 roku obowiązywała nazwa: czarno-biała. 
Statistical analysis showed that the genetic variant of the polymorphic site and the year of analysis had a highly significant effect on the investigated traits. It was shown that for the entire population of cows milk yields were increasing year by year, while in the period of analysis average production progress and cumulative progress amounted to $347.9 \mathrm{~kg}$ and $2435.0 \mathrm{~kg}$ milk, respectively. The greatest annual production progress $(523.9 \mathrm{~kg})$ was recorded in 2005 , when it was $108 \%$ yield from the previous year. Considering the analysed parameters in cows with the identified genetic variant at locus $c .1178 \mathrm{G}>A$, the greatest average annual production progress for milk yield $(375.2 \mathrm{~kg})$ was found for the $A A$ homozygotes, whereas it was lowest $(341.7 \mathrm{~kg})$ for cows with the GG genotype. The greatest annual production progress of $589.8 \mathrm{~kg}$ milk was recorded in 2005 for the GG homozygotes. In turn, the AG heterozygotes had annual progress for that trait ranging from 101.9 to $108 \%$. Analysis of the level of the cumulative progress over the period of 2002-2009 for milk yield in cows varying in their genotypes in the investigated polymorphic site showed its greatest value amounting to $2626.3 \mathrm{~kg}$ milk in the AA homozygotes, followed by the AG heterozygotes $(2495.3 \mathrm{~kg})$ and the GG homozygotes $(2391.8 \mathrm{~kg})$. When investigating average milk yields of cows over the period of the study statistically significant differences were observed in 2007 and 2008 between the genotypes for the discussed parameter. The AA homozygotes were characterised by the lowest milk yields and differed from the other genotypes at the significance levels $P \leq 0.01$ and $P \leq 0.05$ in 2008 and 2007 , respectively.

Table 2 presents results of the calculated production progress for the yield of fat in the years of 2002-2009 for the entire population of Polish Holstein-Friesian Black-and-White cows and in terms of division into genotypes at locus $c .1178 G>A$. Statistical analysis showed a significant effect (at $P \leq 0.01$ ) of genetic variants at locus c.1178G $>A$ and the year of the study on the investigated traits. In the period of analysis in the entire cattle population the average production progress for the yield of milk fat was $13.3 \mathrm{~kg}$, while over several years the greatest value of this parameter $(29.1 \mathrm{~kg})$ was recorded for cows in 2007 . Considering cumulative progress in 2009 production of this milk component increased by $38.8 \%$ in relation to 2002. In terms of the division of cows into genotypes at locus c.1178G>A the greatest value $(14.7 \mathrm{~kg})$ of production progress for the yield of milk fat in the discussed period was found for cows with the AA genotype, followed by the GG $(13.7 \mathrm{~kg})$ and the AG genotype $(12.1 \mathrm{~kg})$. Only in 2004 and 2005 statistically significant differences were observed between the genotypes in terms of the yield of milk fat. In that period cows with the GG genotype were characterised by significantly higher production of milk fat in comparison to the AA homozygotes (at $P \leq 0.01$ ) and the $A G$ heterozygotes (at $P \leq 0.05$ ). Moreover, in 2005 for that milking performance trait cows with the $A G$ genetic variant differed $(P \leq 0.01)$ from the AA homozygotes. When analysing cumulative production progress for the yield of fat its highest value $(102.9 \mathrm{~kg})$ in 2009 was recorded for the AA homozygotes, while it was lowest for the AG heterozygotes $(85 \mathrm{~kg})$, with the increase in milk fat production in relation to 2002 by $44.8 \%$ and $34.7 \%$, respectively.

Statistical analysis showed the effect of genetic variants at locus c.1178G $>A$ and the year of analysis on annual and cumulative production progress in the yield of milk protein (Table 3 ). For the entire population the average annual value of production progress for the production of milk protein was $11.2 \mathrm{~kg}$ and the calculated cumulative progress was $78.3 \mathrm{~kg}$ protein. 
Table 2. Production progress for fat yield [kg] in the years 2002-2009 in the whole studied population of Holstein-Friesian Black-and-White cows ${ }^{a}$ and with regard to genotyping in locus c.1178G>A

Tabela 2. Postęp produkcyjny dla wydajności tłuszczu [kg] w latach 2002-2009 w całej badanej populacji krów rasy polskiej holsztyńsko-fryzyjskiej odmiany czarno-białej ${ }^{a}$, z uwzględnieniem podziału na genotypy w locus c.1178G>A

\begin{tabular}{|c|c|c|c|c|c|c|c|c|c|c|c|c|c|c|c|c|c|c|c|c|}
\hline \multirow{3}{*}{$\begin{array}{c}\text { Year } \\
\text { Rok } \\
* *\end{array}$} & \multirow{2}{*}{\multicolumn{5}{|c|}{$\begin{array}{l}\text { Analysed population } \\
\text { Badana populacja }\end{array}$}} & \multicolumn{15}{|c|}{ Genotype - in locus c.1178G>A - Genotyp w locus c.1178G>A } \\
\hline & & & & & & & & AA ** & & & & & & & & & & GG $* *$ & & \\
\hline & $\bar{x}$ & $x_{i}-x_{i-1}$ & $\%$ & $\mathrm{x}_{\mathrm{i}}-\mathrm{x}_{0}$ & $\%$ & $\bar{x}$ & $\mathrm{x}_{\mathrm{i}}-\mathrm{X}_{\mathrm{i}-1}$ & $\%$ & $\mathrm{x}_{\mathrm{i}}-\mathrm{x}_{0}$ & $\%$ & $\bar{x}$ & $\mathrm{x}_{\mathrm{i}}-\mathrm{x}_{\mathrm{i}-1}$ & $\%$ & $\mathrm{x}_{\mathrm{i}}-\mathrm{x}_{0}$ & $\%$ & $\bar{x}$ & $x_{i}-x_{i-1}$ & $\%$ & $\mathrm{x}_{\mathrm{i}}-\mathrm{x}_{0}$ & $\%$ \\
\hline 2002 & 240.9 & A & & $\begin{array}{l}\text { ABCD } \\
\text { EFG }\end{array}$ & & $\begin{array}{c}229.5 \\
a\end{array}$ & & & $\begin{array}{l}\text { ABC } \\
\text { Dab }\end{array}$ & & $\begin{array}{c}245.3 \\
a\end{array}$ & & & $\begin{array}{l}\text { ABC } \\
\text { DEF }\end{array}$ & & 240.2 & A & & $\begin{array}{l}\text { ABCD } \\
\text { EFG }\end{array}$ & \\
\hline 2003 & 252.7 & $\begin{array}{l}11.8 \\
A B\end{array}$ & 104.9 & $\begin{array}{c}11.8 \\
\mathrm{~A}\end{array}$ & 104.9 & 244.5 & 15.0 & 106.5 & 15.0 & 106.5 & 244.8 & $\begin{array}{c}-0.5 \\
\mathrm{~A}\end{array}$ & 99.8 & -0.5 & 99.8 & 256.4 & $\begin{array}{c}16.2 \\
\mathrm{AB}\end{array}$ & 106.7 & $\begin{array}{c}16.2 \\
\mathrm{~A}\end{array}$ & 106.7 \\
\hline 2004 & 272.3 & $\begin{array}{l}19.6 \\
\mathrm{BC}\end{array}$ & 107.8 & $\begin{array}{c}31.4 \\
\mathrm{~B} \\
\end{array}$ & 113.0 & $\begin{array}{c}261.9 \\
\mathrm{~A}\end{array}$ & $\begin{array}{c}17.4 \\
\mathrm{~A}\end{array}$ & 107.1 & $\begin{array}{c}32.4 \\
\mathrm{a} \\
\end{array}$ & 114.1 & $\begin{array}{c}263.8 \\
a \\
\end{array}$ & $\begin{array}{c}19.0 \\
\mathrm{~A}\end{array}$ & 107.8 & $\begin{array}{c}18.5 \\
\mathrm{~A}\end{array}$ & 107.5 & $\begin{array}{c}276.9 \\
\mathrm{Aa}\end{array}$ & $\begin{array}{c}20.5 \\
\mathrm{Ba}\end{array}$ & 108.0 & $\begin{array}{c}36.7 \\
\mathrm{~B}\end{array}$ & 115.3 \\
\hline 2005 & 280.5 & $\begin{array}{c}8.2 \\
\mathrm{C}\end{array}$ & 108.0 & $\begin{array}{c}39.6 \\
\mathrm{C}\end{array}$ & 116.4 & $\begin{array}{c}257.5 \\
A B\end{array}$ & $\begin{array}{c}-4.4 \\
A B\end{array}$ & 98.3 & $\begin{array}{c}28.0 \\
b\end{array}$ & 112.2 & $\begin{array}{c}272.9 \\
\mathrm{Ba}\end{array}$ & 9.1 & 103.4 & $\begin{array}{c}27.6 \\
\text { B }\end{array}$ & 111.3 & $\begin{array}{c}285.5 \\
\mathrm{Aa}\end{array}$ & $\begin{array}{l}8.6 \\
a \\
\end{array}$ & 103.1 & $\begin{array}{c}45.3 \\
\mathrm{C}\end{array}$ & 118.6 \\
\hline 2006 & 282.9 & $\begin{array}{c}2.4 \\
\mathrm{D}\end{array}$ & 103.0 & $\begin{array}{l}42.0 \\
\mathrm{D}\end{array}$ & 117.4 & 283.6 & $\begin{array}{c}26.1 \\
\mathrm{~B} \\
\end{array}$ & 110.1 & $\begin{array}{c}54.1 \\
\mathrm{~A}\end{array}$ & 123.6 & 279.3 & $\begin{array}{c}6.4 \\
\mathrm{~B}\end{array}$ & 102.3 & $\begin{array}{c}34.0 \\
\mathrm{C}\end{array}$ & 113.9 & 284.7 & $\begin{array}{l}-0.8 \\
\mathrm{C}\end{array}$ & 99.7 & $\begin{array}{c}44.5 \\
D\end{array}$ & 118.5 \\
\hline 2007 & 312.0 & $\begin{array}{l}29.1 \\
\mathrm{DE}\end{array}$ & 110.3 & $\begin{array}{c}71.1 \\
\mathrm{E}\end{array}$ & 129.5 & 304.1 & 20.5 & 107.2 & $\begin{array}{c}74.6 \\
B\end{array}$ & 132.5 & 307.8 & $\begin{array}{l}28.5 \\
\mathrm{BC}\end{array}$ & 110.2 & $\begin{array}{c}62.5 \\
D\end{array}$ & 125.5 & 314.4 & $\begin{array}{c}29.7 \\
C D\end{array}$ & 110.4 & $\begin{array}{c}74.2 \\
\mathrm{E}\end{array}$ & 130.9 \\
\hline 2008 & 329.3 & $\begin{array}{c}17.3 \\
E\end{array}$ & 105.5 & $\begin{array}{c}88.4 \\
F\end{array}$ & 136.7 & 326.7 & $\begin{array}{c}22.6 \\
\mathrm{C}\end{array}$ & 107.4 & $\begin{array}{c}97.2 \\
\mathrm{C}\end{array}$ & 142.3 & 323.0 & $\begin{array}{c}15.2 \\
\mathrm{C}\end{array}$ & 104.9 & $\begin{array}{c}77.7 \\
\mathrm{E}\end{array}$ & 131.7 & 332.6 & $\begin{array}{c}18.2 \\
\mathrm{D}\end{array}$ & 105.8 & $\begin{array}{c}92.4 \\
\mathrm{~F}\end{array}$ & 138.5 \\
\hline 2009 & 334.3 & 5.0 & 101.5 & $\begin{array}{c}93.4 \\
G\end{array}$ & 138.8 & 332.4 & $\begin{array}{l}5.7 \\
\mathrm{C}\end{array}$ & 101.7 & $\begin{array}{c}102.9 \\
\mathrm{D}\end{array}$ & 144.8 & 330.3 & 7.3 & 102.3 & $\begin{array}{c}85.0 \\
F\end{array}$ & 134.7 & 336.2 & 3.6 & 101.1 & $\begin{array}{c}96.0 \\
G\end{array}$ & 140.0 \\
\hline $\bar{x}$ & & 13.3 & & & & & 14.7 & & & & & 12.1 & & & & & 13.7 & & & \\
\hline
\end{tabular}

Explanations see Table 1 - Objaśnienia zob. tab. 1. 
Table 3. Production progress for protein yield [kg] in the years 2002-2009 in the whole studied population of Holstein-Friesian Black-and-White cows and with regard to genotyping in locus c.1178G>A

Tabela 3. Postęp produkcyjny dla wydajności białka [kg] w latach 2002-2009 w całej badanej populacji krów rasy polskiej holsztyńsko-fryzyjskiej odmiany czarno-białej ${ }^{a}$, z uwzględnieniem podziału na genotypy w locus c.1178G>A

\begin{tabular}{|c|c|c|c|c|c|c|c|c|c|c|c|c|c|c|c|c|c|c|c|c|}
\hline \multirow{3}{*}{$\begin{array}{c}\text { Year } \\
\text { Rok } \\
* *\end{array}$} & \multirow{2}{*}{\multicolumn{5}{|c|}{$\begin{array}{l}\text { Analysed population } \\
\text { Badana populacja }\end{array}$}} & \multicolumn{15}{|c|}{ Genotype - in locus c.1178G>A - Genotyp w locus c.1178G>A } \\
\hline & & & & & & \multicolumn{5}{|c|}{ AA $* *$} & \multicolumn{5}{|c|}{ AG $* *$} & \multicolumn{5}{|c|}{ GG ** } \\
\hline & $\bar{x}$ & $x_{i}-x_{i-1}$ & $\%$ & $\mathrm{x}_{\mathrm{i}}-\mathrm{x}_{0}$ & $\%$ & $\bar{x}$ & $x_{i}-x_{i-1}$ & $\%$ & $x_{i}-x_{0}$ & $\%$ & $\bar{x}$ & $x_{i}-x_{i-1}$ & $\%$ & $x_{i}-x_{0}$ & $\%$ & $\bar{x}$ & $x_{i}-x_{i-1}$ & $\%$ & $x_{i}-x_{0}$ & $\%$ \\
\hline 2002 & 193.0 & A & & $\begin{array}{l}A B C D \\
E F G\end{array}$ & & $\begin{array}{c}185.9 \\
\mathrm{Aa}\end{array}$ & A & & $\begin{array}{l}\text { ABC } \\
\text { DEFG }\end{array}$ & & $\begin{array}{c}195.2 \\
A\end{array}$ & & & $\begin{array}{l}A B C \\
D E F\end{array}$ & & $\begin{array}{l}192.7 \\
a\end{array}$ & A & & $\begin{array}{c}\text { ABC } \\
\text { DEF } \\
G\end{array}$ & \\
\hline 2003 & 203.5 & $\begin{array}{l}10.5 \\
A B\end{array}$ & 105.4 & $\begin{array}{c}10.5 \\
\mathrm{~A}\end{array}$ & 105.4 & $\begin{array}{c}199.8 \\
a \\
\end{array}$ & $\begin{array}{c}13.9 \\
A B\end{array}$ & 107.5 & $\begin{array}{c}13.9 \\
\mathrm{~A}\end{array}$ & 107.5 & $\begin{array}{c}198.2 \\
\mathrm{~b}\end{array}$ & $\begin{array}{c}3.0 \\
\mathrm{~A}\end{array}$ & 101.5 & 3.0 & 101.5 & $\begin{array}{l}205.9 \\
a b\end{array}$ & $\begin{array}{c}13.2 \\
A B\end{array}$ & 106.9 & $\begin{array}{c}13.2 \\
\mathrm{~A}\end{array}$ & 106.9 \\
\hline 2004 & 223.6 & $\begin{array}{l}20.6 \\
\mathrm{BC}\end{array}$ & 109.9 & $\begin{array}{c}30.6 \\
\mathrm{~B}\end{array}$ & 115.9 & 223.0 & $\begin{array}{c}23.2 \\
B\end{array}$ & 111.6 & $\begin{array}{c}37.1 \\
B\end{array}$ & 120.0 & $\begin{array}{c}218.6 \\
A\end{array}$ & $\begin{array}{c}20.4 \\
A B\end{array}$ & 110.3 & $\begin{array}{c}23.4 \\
\mathrm{~A}\end{array}$ & 112.0 & $\begin{array}{c}225.9 \\
\mathrm{~A}\end{array}$ & $\begin{array}{l}20.0 \\
\mathrm{BC}\end{array}$ & 109.7 & $\begin{array}{c}33.2 \\
\mathrm{~B}\end{array}$ & 117.2 \\
\hline 2005 & 237.1 & $\begin{array}{c}13.4 \\
\mathrm{C}\end{array}$ & 106.0 & $\begin{array}{c}44.1 \\
\mathrm{C}\end{array}$ & 122.8 & $\begin{array}{c}227.5 \\
\mathrm{~A}\end{array}$ & $\begin{array}{c}4.5 \\
\mathrm{C}\end{array}$ & 102.0 & $\begin{array}{c}41.6 \\
C\end{array}$ & 122.4 & $\begin{array}{c}231.1 \\
B\end{array}$ & $\begin{array}{l}12.5 \\
\mathrm{BC}\end{array}$ & 105.7 & $\begin{array}{c}35.9 \\
B\end{array}$ & 118.4 & $\begin{array}{c}240.3 \\
\mathrm{AB}\end{array}$ & $\begin{array}{c}14.4 \\
\mathrm{C}\end{array}$ & 106.4 & $\begin{array}{c}47.6 \\
C\end{array}$ & 124.7 \\
\hline 2006 & 238.7 & $\begin{array}{l}1.7 \\
D\end{array}$ & 100.7 & $\begin{array}{l}45.7 \\
D\end{array}$ & 123.7 & $\begin{array}{c}243.6 \\
a b\end{array}$ & $\begin{array}{c}16.1 \\
C\end{array}$ & 107.1 & $\begin{array}{c}57.7 \\
D\end{array}$ & 131.0 & $\begin{array}{l}238.0 \\
a\end{array}$ & $\begin{array}{l}6.9 \\
C D\end{array}$ & 103.0 & $\begin{array}{c}42.8 \\
C\end{array}$ & 121.9 & $\begin{array}{c}238.8 \\
b\end{array}$ & $\begin{array}{l}-1.5 \\
D\end{array}$ & 99.4 & $\begin{array}{c}46.1 \\
D\end{array}$ & 123.9 \\
\hline 2007 & 253.3 & $\begin{array}{l}14.6 \\
\mathrm{DE}\end{array}$ & 106.1 & $\begin{array}{c}60.3 \\
E\end{array}$ & 131.2 & $\begin{array}{c}242.5 \\
\mathrm{Aa}\end{array}$ & $\begin{array}{c}-1.1 \\
a \\
\end{array}$ & 99.5 & $\begin{array}{c}56.6 \\
E\end{array}$ & 130.5 & $\begin{array}{c}255.8 \\
\mathrm{~A} \\
\end{array}$ & $\begin{array}{l}17.8 \\
\mathrm{DE}\end{array}$ & 107.5 & $\begin{array}{c}60.6 \\
D\end{array}$ & 131.0 & $\begin{array}{c}252.6 \\
a \\
\end{array}$ & $\begin{array}{l}13.8 \\
\mathrm{DE}\end{array}$ & 105.8 & $\begin{array}{c}59.9 \\
E\end{array}$ & 131.1 \\
\hline 2008 & 268.5 & $15.2 \mathrm{E}$ & 106.0 & $\begin{array}{c}75.5 \\
F\end{array}$ & 139.1 & $\begin{array}{c}251.9 \\
A B\end{array}$ & $\begin{array}{l}9.4 \\
\mathrm{Da}\end{array}$ & 103.9 & $\begin{array}{c}66.0 \\
F\end{array}$ & 135.5 & $\begin{array}{c}267.6 \\
\text { A }\end{array}$ & $\begin{array}{c}11.8 \\
\mathrm{E}\end{array}$ & 104.6 & $\begin{array}{c}72.4 \\
\mathrm{E}\end{array}$ & 137.1 & $\begin{array}{c}270.4 \\
\text { B }\end{array}$ & $\begin{array}{c}17.8 \\
\mathrm{E}\end{array}$ & 107.0 & $\begin{array}{c}77.7 \\
F\end{array}$ & 140.3 \\
\hline 2009 & 271.3 & 2.8 & 101.0 & $\begin{array}{c}78.3 \\
G\end{array}$ & 140.6 & 263.6 & $\begin{array}{c}11.7 \\
\mathrm{D}\end{array}$ & 104.6 & $\begin{array}{c}77.7 \\
\mathrm{G}\end{array}$ & 141.8 & 267.5 & -0.1 & 99.9 & $\begin{array}{c}72.3 \\
F \\
\end{array}$ & 137.0 & 273.5 & 3.1 & 101.1 & $\begin{array}{c}80.8 \\
G\end{array}$ & 141.9 \\
\hline $\bar{x}$ & & 11.2 & & & & & 11.1 & & & & & 10.3 & & & & & 11.5 & & & \\
\hline
\end{tabular}

Explanations see Table 1 - Objaśnienia zob. tab. 1. 
Analysis of annual production progress for the yield of milk protein in individual years showed its highest value irrespective of genotype in 2004. The percentage value of the yield of milk protein in relation to the previous year ranged from 109.7 for the $G G$ homozygotes to 111.6 for the AA homozygotes. The greatest average annual production progress for this parameter $(11.5 \mathrm{~kg})$ was recorded for the GG homozygotes, while it was lowest $(10.3 \mathrm{~kg})$ in the $A G$ heterozygotes. Cows with the $A A, A G$ and $G G$ genetic variants in the analysed period had cumulative production progress for the yield of milk protein amounting to $77.7 \mathrm{~kg}, 72.3$ $\mathrm{kg}$ and $80.8 \mathrm{~kg}$, respectively. In terms of the average yields of milk protein in individual years the greatest value of that trait was recorded for the GG homozygous cows. In turn, cows with the AA genotype at locus $c .1178 \mathrm{G}>A$ had the lowest yield of milk protein $(185.9 \mathrm{~kg}$ ) in 2002 , which most probably influenced the cumulative production progress in the investigated period.

\section{DISCUSSION}

The positive effect of the AA genetic variant on the yields of milk, milk fat and protein was reported by Parmentier et al. (2001). In turn, Renaville et al. (1997) recorded an advantageous dependence between the $A A$ variant and the yield of milk and protein. Similar dependencies between the AA genotype at locus c.1178G $>A$ and milk production were shown by Hori-Oshima and Barreas-Serrano (2004), Vargas et al. (2004) and Doosti et al. (2011). In contrast, opposite results were given by Oprządek et al. (2006), who reported the lowest milk yield in the 2nd lactations and the lowest yields of milk fat and protein in the 2nd and 3rd lactations for cows with the AA genotype.

A greater production progress and cumulative progress in the investigated period (except for production progress for milk yield in 2006 and 2009) were found for cows in this study in comparison to the active population. Yaeghoobi et al. (2011) showed that over a period of 10 years in primiparous Holstein cows the annual phenotypic trend for milk yield was $71.99 \mathrm{~kg}$. In a study by Roman et al. (1999) conducted on Jersey cows it was found that annual changes in genetic progress for milk yield ranged from 36.8 to $41.0 \mathrm{~kg} / \mathrm{head}$. In turn, Neja et al. (2013) reported that in the period from 2001 to 2009 in Black-and-White cows and Polish Holstein-Friesian Black-and-White cows in Poland, covered by performance testing, the cumulative production progress for milk yield was $1423 \mathrm{~kg}$. Results for analyses in the groups of animals varying in their genotype at locus $c .1178 \mathrm{G}>A$ in comparison to the results published by KCHZ (2003, 2004, 2005) and PFHBiPM (2006, 2007, 2008, 2009, 2010) concerning the entire active population of cows in Poland and (apart from very few cases) showed in the cows included in this study greater milk yields as well as more advantageous annual and cumulative production progress in milk yields.

Analysis of results recorded in this study showed that a greater yield of milk fat, greater annual production progress (except for 2005 and 2009) as well as a more advantageous cumulative production progress in the investigated period for animals included in this study in comparison to the entire active population of cows in Poland, which productivity was presented in studies published by $\operatorname{KCHZ}(2003,2004,2005)$ and PFHBiPM $(2006,2007$, 2008, 2009, 2010). In turn, Neja et al. (2013) reported that in the population of the investigated cattle breeds, i.e. Black-and-White and Polish Holstein-Friesian Black-and-White, in the 
period of 2001-2009 cumulative production progress in the yield of milk fat was $57 \mathrm{~kg}$. The value of this parameter in comparison to the results obtained in this study for the period of 2002-2009 was by $24.6 \mathrm{~kg}$ lower. A comparison of results for animals differing in their genotype at locus $c .1178 \mathrm{G}>A$ to those for the entire active population of cows in the investigated period indicates that among the three genetic variants the least advantageous values of the analysed traits are found for the AG heterozygotes.

When comparing results calculated for the entire population of cows with those published by $\operatorname{KCHZ}(2003,2004,2005)$ and PFHBiPM $(2006,2007,2008,2009,2010)$ for the entire active population in Poland we may state that for the animals analysed in this study for all the investigated traits higher values were recorded in 2006 (except for annual production progress in the yield of milk protein). In their study covering a 10-year period Yaeghoobi et al. (2011) stated in primiparous Holstein cows an annual phenotypic trend for the yield of milk protein of $1.4 \mathrm{~kg}$. In turn, Neja et al. (2013) reported that in the active population of Black-and-White and Polish Holstein-Friesian cows in the period from 2001 to 2009 cumulative production progress for the yield of milk protein was $48 \mathrm{~kg}$. A comparison of results for animals differing in their genotype at the analysed locus with those published by $\mathrm{KCHZ}$ (2003, 2004, 2005) and PFHBiPM $(2006,2007,2008,2009,2010)$ for the entire Polish active population showed the most advantageous values of milk protein parameters for cows with the GG genotype.

\section{CONCLUSION}

Polish Holstein-Friesian Black-and-White cows with the AA genotype at locus c.1178G $>A$ were characterised by the greatest annual production progress and cumulative progress for the yield of milk and the yield of milk fat. In turn, in terms of the yield of milk protein more advantageous values of calculated parameters were found for the GG homozygotes.

\section{REFERENCES}

Atashi H., Moradi Shahrbabak M., Abdolmohammadi A. 2006. Study of some suggest measures of milk yield persistency and their relationships. Int. J. Agric. Biol. 8, 387-390.

Augustijn K.D., DuvaL D.L., Wechselberger R., Kaptein R., Gutierrez-Hartmann A., Van Der Viet P.C. 2002. Structural characterization of the PIT-1/ETS-1 interaction: PIT-1 phosphorylation regulates PIT-1/ETS-1 binding. Proceedings of the National Academy of Sciences of the United States of America. 99, 12657-12662.

Bodner M., Castrillo J.L., Theill L.E., Deerinck T., Ellisman M., Karin M. 1988. The pituitaryspecyfictranscription factors GHF-1 is a homeobox-containing protein. Cell 55, 505-518.

Doosti A., Arshi A., Momenl B. 2011. Molecular study of PIT1 gene polymorphism in Holstein and Iranian native cattle. Afr. J. Agric. Res. 6, 4467-4470.

Georges M., Nielsen D., Mackinnon M., Mishra A., Okimoto R., Pasquino A.T., Sargeant L.S., Sorensen A., Steele M.R., Zhao X., Womack J.E., Hoeschele I. 1995. Mapping quantitative trait loci controlling milk production in dairy cattle by exploiting progeny testing. Genetics 139, 907-920.

Hori-Oshima S., Barreas-Serrano A. 2004. Relationships between DGAT1 and PIT-1 genes polymorphism and milk yield in Holstein cattle. J. Anim. Sci. 81(Suppl.1), 282.

KCHZ. 2003. Ocena wartości użytkowej krów mlecznych. Wyniki za 2002 rok. Warszawa, Wydaw. $\mathrm{KCHZ}$. [in Polish] 
KCHZ. 2004. Ocena wartości użytkowej krów mlecznych. Wyniki za 2003 rok. Warszawa, Wydaw. $\mathrm{KCHZ}$. [in Polish]

KCHZ. 2005. Ocena wartości użytkowej krów mlecznych. Wyniki za 2004 rok. Warszawa, Wydaw. $\mathrm{KCHZ}$. [in Polish]

Lin S.C., Li S., Drolet D.W., Rosenfeld M.G. 1994. Pituitary ontogeny of the snell dwarf mousereveals Pit-1 independent and Pit-1 dependent origins of the thyrotrope. Development 120, 515-522.

Neja W., Jankowska M., Sawa A., Bogucki M. 2013. Analiza użytkowości mlecznej i rozpłodowej krów krajowej populacji aktywnej [Analysis of milk and reproductive performance of the active population of cows in Poland]. JCEA 14, 91-101. [in Polish]

Nelson C., Albert V.R., Elsholtz H.P., Lu L.I., Rosenfeld M.G. 1988. Activation of cell - specific expression of rat growth hormone and prolactin gene by a common transcription factor. Science $239,1400-1405$.

Oprządek J., Dymnicki E., Oprządek A., Zwierzchowski L. 2006. Zależności pomiędzy polimorfizmem wybranych genów a użytkowością mleczną, długością okresu międzyocieleniowego, wskaźnikiem inseminacji krów rasy czarno-białej. Szczecin, Wydaw. AR, 28-37. [in Polish]

Parmentier I., Gengler N., Fontaine S., Auvray B., Burnside T., Portetelle D., Renaville R. 2001. PIT-1 a candidate gene for mass assisted selection in dairy bulls. J. Anim. Sci. 79(Suppl. 1), 340.

PFHBiPM. 2006. Ocena i hodowla bydła mlecznego. Dane za 2005 rok. Warszawa, Wydaw. PFHBiPM. [in Polish]

PFHBiPM. 2007. Ocena i hodowla bydła mlecznego. Dane za 2006 rok. Warszawa, Wydaw. PFHBiPM. [in Polish]

PFHBiPM. 2008. Ocena i hodowla bydła mlecznego. Dane za 2007 rok. Warszawa, Wydaw. PFHBiPM. [in Polish]

PFHBiPM. 2009. Ocena i hodowla bydła mlecznego. Dane za 2008 rok. Warszawa, Wydaw. PFHBiPM. [in Polish]

PFHBiPM. 2010. Ocena i hodowla bydła mlecznego. Dane za 2009 rok. Warszawa, Wydaw. PFHBiPM. [in Polish]

Renaville R., Gengler N., Vrech E., Prandl A., Massart S., Corradini C., Bertozzi C., Mortiau X.F., Burny A., Portetelle D. 1997. Pit-1 gene polymorphism milk yield and conformation traits for Italian Holstein-Friesian bulls. J. Dairy Sci. 80, 3431-3438.

Rhodes S.J., Chen R., Di Mattia G.E., Scully K.M., Kalla K.A., Lin S.C., Yu V.C., Rosenfeld M.G. 1993. A tissue - specific enhancer confers Pit-1 dependent morphogen inducibility and autoregulation autoregulation on the Pit-1 gene. Gene Dev. 7, 913-932.

Roman R.M., Wilcox C.J., Little R.C. 1999. Genetic trend for milk yield of Jersey and correlated changes in productive and reproductive performance. J. Dairy Sci. 82, 196-204.

SAS ${ }^{\circledR}$ user's guide. 2011. Statistics. Version 9.2. SAS Inst., Cary, NC.

Stancekova K., Vasicek D., Peskovicova D., Bulla J., Kubek A. 1999. Effect of genetics variability of the porcine pituitary-specific transcription factor (Pit-1) on carcass traits in pigs. Anim. Genet. 30, 313-315.

Vargas L.D., Gana E.V., Escudero F.I. 2004. Polomorfismo del gen PIT-1 en vacas lechers de Chile Central. [Pit-1 gene polymorphism in dairy cows from Central Chile]. Arch. Zootech. 53, 217-220. [in Spain]

Woollard J., Schmitz C.B., Freeman A.E., Tuggle C.K. 1994. Rapid communication: Hinf polymorphism at the bovine Pit-1 locus. J. Anim. Sci. 72, 3267-3267.

Woollard J., Tuggle C.K., Ponce De León F.A. 2000. Rapid communication: Localization of PU1F1 to bovine, ovine and caprine 1q21-22. J. Anim. Sci. 78, 242-243.

Yaeghoobi R., Doosti A., Noorian A.M., Bahrami A.M. 2011. Genetic parameters and trends of milk and fat yield in Holstein's dairy cattle of West Provinces of Iran. Int. J. Dairy Sci. 6, 142-149. 
Abstract. The aim of this study was to analyse the dependence between PIT-1 gene polymorphism in exon 6 of bovine chromosome 1 (c.1178G>A) and the level of production progress in Polish Holstein-Friesian Black-and-White cows. The greatest annual production progress and cumulative progress for the yield of milk and milk fat was found for the $A A$ homozygotes. For the yield of milk protein more advantageous values of calculated parameters were recorded for the GG homozygotes. 
